\title{
Distribuição de Conteúdo via Conexão 5G Híbrida Terrestre/Satélite
}

\author{
Tibério Tavares Rezende ${ }^{1}$, Victor Hugo D. D’Ávila ${ }^{1}$, \\ Karine L. M. da Costa ${ }^{1}$ e Antônio Marcos Alberti ${ }^{1}$ \\ ${ }^{1}$ ICT Lab - Instituto Nacional de Telecomunicações (INATEL) \\ Caixa Postal: 37.540.000 - Santa Rita do Sapucaí MG - Brasil \\ (tiberio; victorhugo; karine.costa) @mtel.inatel.br, \\ alberti@inatel.br
}

\begin{abstract}
This work proposes a hybrid $5 G$ terrestrial-satellite architecture for a cache-based content distribution application. The article presents the selected technologies, their configurations in a real environment, performance analysis and lessons learned. For the experimental environment, Inatel $5 G$ Modems and SES-14 satellite with COMTECH CDM-760 modem were used. The results show that the use of network caches brings great benefit to content distribution in $5 G$ terrestrial/satellite hybrid.
\end{abstract}

Resumo. Esse trabalho propõem uma arquitetura híbrida $5 G$ terrestre/satélite para a distribuição de conteúdos com cache de rede. $O$ artigo apresenta as tecnologias selecionadas, suas configurações em ambiente real, análise de desempenho e lições aprendidas. Para o ambiente experimental, foram utilizados Modems $5 G$ do Inatel e satélite SES-14 com modem COMTECH CDM-760. Os resultados mostram que o uso de cache traz grande benefício a distribuição de conteúdos em $5 G$ híbrido terrestre/satélite.

\section{Introdução}

Os sistemas de comunicação móvel evoluíram através de uma série de gerações. De sistemas analógicos (1G), passando pelo Global System for Mobile Communications (GSM) (2G), pelo International Mobile Telecommunications-2000 (IMT-2000) (3G), para os sistemas Long Term Evolution-Advanced (LTE-A) (4G), que são os mais utilizados atualmente. De acordo com [Evans 2015], os sistemas de comunicações móveis via satélite se desenvolveram de forma independente dos sistemas terrestres por um longo período. No entanto, em vários parâmetros, como, área de cobertura e taxas de dados, os sistemas de comunicação móvel via satélite e terrestres se mostram complementares [J. Min 2016]. O futuro das redes sem fio é uma arquitetura de rede na qual as informações podem ser compartilhadas e acessíveis em qualquer lugar do mundo e em qualquer momento, de maneira transparente, sem interrupções. Atualmente, redes móveis têm sido amplamente utilizadas em aplicações de comunicação Machine to Machine (M2M) e Internet of Things (IoT). Ao oferecer recursos necessários para atingir a conectividade ubíqua, a comunicação via satélite torna-se um elemento chave para este cenário. Isto se deve ao fato das particularidades únicas apresentadas pela comunicação via satélite em frente aos desafios de conectividade, como por exemplo, limitações de cobertura presentes nas redes móveis atuais, principalmente quando se trata de áreas mais afastadas. Nesse contexto, 
órgãos padronizadores dos sistemas de telecomunicações móveis e via satélite têm unido esforços para oferecer serviços de alta qualidade e disponibilidade no cenário M2M/IoT.

De acordo com documentos do International Telecommunication Union - Radiocommunication (ITU-R) e do International Mobile Telecommunications (IMT), o objetivo do 5G (Quinta Geração de Comunicação Sem Fio Móvel) é viabilizar uma sociedade conectada de forma integrada a partir de 2020, possibilitando reunir pessoas, objetos, dados, aplicações, sistemas de transporte e cidades em um ambiente de comunicações inteligente [Marcus 2015], [Dai 2018], [C. Young 2016] e [IEEE 2016]. O desenvolvimento do 5G terrestre tem sido o principal agente para revolucionar as comunicações móveis via satélite [A. Guidotti 2018]. O sistema de comunicação híbrido satélite-terrestre, e em especial, a integração de comunicações 5G via satélite e terrestre, é considerada uma tendência de desenvolvimento futuro [K. Liolis 2018].

Esse trabalho propõem uma arquitetura híbrida terrestre/satélite para infraestrutura da rede $5 \mathrm{G}$ e aplica a solução obtida na distribuição de conteúdo com cache de rede. A arquitetura trata de 5G híbrido com armazenamento temporário na própria rede. A proposta se baseia nos principais projetos do programa Horizon 2020 e nos órgãos padronizadores, tais como 3rd Generation Partnership Project (3GPP), European Telecommunications Standards Institute (ETSI) e ITU-R. Conceitos como controle de rede, segurança, redes centradas em informação, virtualização, distribuição de conteúdo e novos meios de comunicação são explorados e correlacionados. Construímos uma Content Distribution Network (CDN) privada em laboratório para operar via enlace $5 \mathrm{G}$ usando softwares Open Source e prontamente disponíveis. Configuramos e testamos essa rede com equipamentos reais das empresas de soluções para satélite, SES-14 e COMTECH, ambas parceiras do Projeto B financiado pelo Sindicato Nacional das Empresas de Telecomunicações por Satélite (SINDISAT) em parceria com o Instituto Nacional de Telecomunicações (Inatel). Os resultados obtidos mostram claramente a vantagem do uso de cache de rede para distribuir conteúdos em 5G híbrido. O restante do artigo está organizado como segue. Os principais trabalhos e projetos relacionados com a evolução da comunicação via satélite em sistemas de comunicações móveis 5G são abordados na Seção 2. A Seção 3 apresenta uma proposta de arquitetura de rede 5G híbrida centrada na distribuição de conteúdo e construída no ambiente de testes do Information and Communications Technologies Laboratory (ICT Lab), do Inatel. Com a pretensão de contribuir para a evolução do 5G no Brasil, a Seção 4 apresenta os principais resultados obtidos com os testes e a Seção 5 apresenta as conclusões sobre os trabalhos efetuados.

\section{Redes Móveis 5G operando via Satélite}

Essa seção descreve os trabalhos e projetos mais relevantes relacionados com redes 5G híbridas até o momento de escrita desse artigo. Considerando que o avanço tecnológico exponencial gera vários efeitos sobre os atuais cenários de informação e tecnologia, nesse contexto, a comunicação via satélite deve ser exposta sempre como uma possibilidade chave, visando atender os mais diversos casos de uso no 5G [A. Guidotti 2018], [W. Ejaz 2016], [Naser 2017]. Esse tipo de tecnologia desempenhará um papel importante ao lado das tecnologias terrestres existentes.

O uso da comunicação via satélite em conjunto com tecnologias de fornecimento de informação e distribuição de conteúdo visando estabelecer comunicação entre as redes 
de acesso, núcleo e de dados no 5G, é uma oportunidade única da atual conjuntura de convergência tecnológica exponencial. Diversas soluções de conectividade e gerenciamento vêm surgindo no intuito de integrar redes terrestres e satelitais e esforços são unidos a fim de alcançar sua interoperabilidade [Y. Kawamoto 2013]. Somam-se aí as oportunidades trazidas pela $\mathrm{M} 2 \mathrm{M} / \mathrm{IoT}$ no ambiente $5 \mathrm{G}$, bem como vários outros cenários importantes para as redes híbridas no Brasil, tais como: (i) broadband móvel extremo, também conhecido como Bitpipe; (ii) serviços de missão crítica de alta confiabilidade e de baixa latência ou Tactile Internet; e (iii) atendimento a áreas remotas, o Wireless Rural Area Network (WRAN). A rede 5G está prevista para garantir a onipresença de dispositivos M2M e IoT para que novos serviços sejam eficientemente suportados [3GPP 2016].

Um projeto que trata especificamente de entrega de conteúdo em $5 \mathrm{G}$ e satélite é o Satellite and Terrestrial Network for 5G (SaT5G) do programa H2020 [SaT5G 2018]. O objetivo do projeto é definir soluções de backhaul e de descarregamento de tráfego baseadas em satélite para uma rede 5G híbrida. O seu escopo cobre tecnologias 5G para obter o melhor valor dos recursos de satélite. Oferece recursos de multicast para entrega de conteúdo, funções de rede virtualizada e propostas para atenuação de latência durante a conectividade. Nesse contexto, a tecnologia de comunicação via satélite deve ser exposta sempre como uma possibilidade chave, visando atender os mais diversos cenários de uso no $5 \mathrm{G}$.

Os satélites têm outras funções importantes no 5G, incluindo o descarregamento de redes terrestres e o fornecimento de comunicações de emergência confiáveis [N. Wang 2019]. No trabalho de [N. Wang 2019] é introduzido uma arquitetura de rede orientada a $5 \mathrm{G}$ que é baseada em comunicações por satélite e computação de borda de acesso múltiplo para dar suporte a aplicativos eMBB, que é investigada na Fase 2 do $5 G$ Infrastructure Public Private Partnership (5GPPP). Os autores estudam técnicas para garantir a eficácia da entrega de arquivos e Quality of Experience (QoE) através da avaliação de desempenho em um enlace real de backhaul de satélite em uma ampla variedade de cenários práticos. Também fornecem insights práticos sobre o comportamento de TCP em um backhaul de satélite em cenários de transmissão ao vivo. O foco é a garantia da QoE dos usuários de streaming ao vivo baseados em HTTP, utilizando os enlaces de satélite, onde a estratégia principal é realizar retenção e localização transitórias de segmentos HTTP MPEG-DASH ou HTTPlive streaming de vídeo no 5G. Pela primeira vez na literatura, os autores realizaram experimentos sobre o desempenho de transmissões de vídeo com resolução $4 \mathrm{~K}$ ao vivo em uma rede principal 5G suportada por um backhaul de satélite geoestacionário.

O objetivo do $5 \mathrm{G}$ é oferecer suporte a vários cenários de uso, como enhanced Mobile Broadband (eMBB), Ultra Reliable Low Latency Communications (URLLC) e massive Machine Type Communications (mMTC) [3GPP 2016]. Nesses cenários, as comunicações via satélite serão usadas para ampliar a cobertura de redes terrestres e fornecer backhaul para redes 5G. A convergência das plataformas de satélite e tecnologias emergentes de fornecimento de conteúdo para redes $5 \mathrm{G}$ representa uma grande oportunidade para a melhoria dos serviços integrados suportados de uma maneira dupla: aumentando a capacidade geral e introduzindo recursos específicos de satélite, potencializados pela CDN, como a grande cobertura geográfica e transmissão nativa de broadcast, multicast ou unicast [M. Luglio 2019]. As plataformas de satélite podem desempenhar um 
papel fundamental nas redes futuras. Essas plataformas podem operar como suporte de serviços como o de cache local assistido, que por sua vez deve representar um recurso essencial da funcionalidade futura da Edge e Cloud Computing [Frangoudis 2017]. O fornecimento de conteúdo multimídia por sistemas de satélite é considerado um serviço promissor em redes emergentes de 5G [N. Panwar 2016]. Para a entrega de conteúdo, a rede CDN é utilizada como um conjunto colaborativo de elementos de rede distribuídos pela Internet, com o objetivo de apoiar a replicação de conteúdo em vários servidores espelhados através do cache. Segundo o trabalho de [Yang 2018], a rede CDN baseada em comunicação via satélite $5 \mathrm{G}$ é uma proposta para combater o congestionamento de entrega de arquivos pela rede terrestre sendo mais escalável e confiável.

Esse artigo sugere uma arquitetura para a distribuição de conteúdo via conexão $5 \mathrm{G}$ híbrida construída com equipamentos reais e descreve em detalhes os procedimentos metodológicos adotados na elaboração da arquitetura, algo ainda não visto na literatura. A arquitetura faz uso de tecnologias e técnicas atuais para melhorar o desempenho de distribuição de conteúdo. Para os testes foram utilizados equipamentos das empresas de soluções para satélite, SES-14, COMTECH e os Modems 5G do Inatel. O enlace 5G é formado por dois modems construídos com rádios Multiple-Input/Multiple-Output (MIMO) operando com a modulação Generalized Frequency Division Multiplexing (GFDM) para a faixa de frequência própria para redes $5 \mathrm{G}$ de longa distância.

\section{Proposta de Arquitetura 5G Híbrida para Distribuição de Conteúdos}

Nessa seção apresentamos uma arquitetura de rede 5G híbrida para distribuição de conteúdo com cache de rede utilizando satélite real e um par de Transceptores Flexíveis MIMO-GFDM (Modems 5G). A arquitetura é formada por nós conectados via enlace de satélite geoestacionário e enlace terrestre que conectam a rede híbrida com recursos virtualizados e CDN para Broadcast e Multicast.

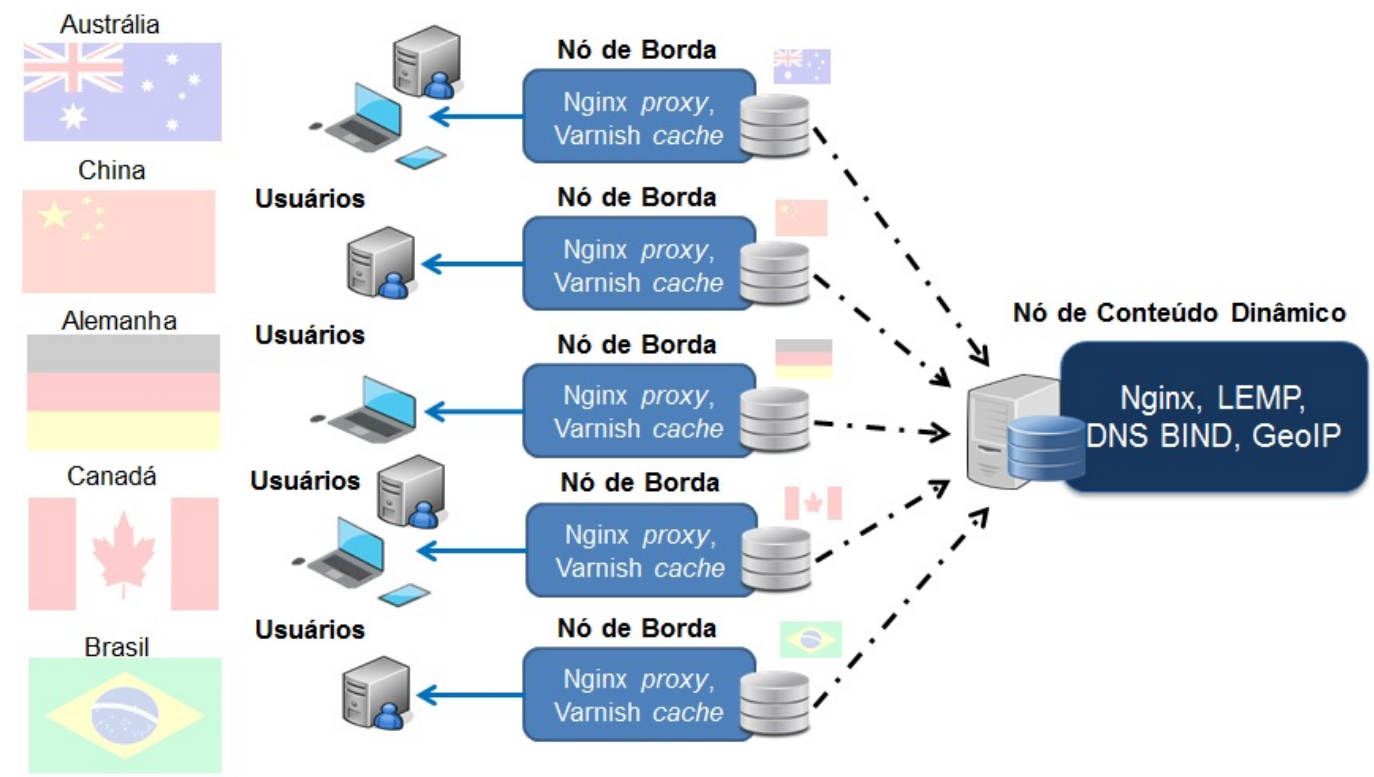

Figura 1. Layout lógico com os nós que formam uma CDN.

A Figura 1 ilustra um layout lógico de uma CDN construída com os componentes escolhidos na arquitetura proposta e mostra usuários em diversos lugares recebendo 
conteúdos do nó de borda que executa os programas Nginx e Varnish. Os nós de borda (edgecdn_node) podem estar localizados geograficamente em qualquer local e podem ser adicionados conforme necessidade. O nó de borda recebe os dados originais do nó de conteúdo dinâmico e armazena uma cópia no local. O nó de conteúdo dinâmico (centralcdn_node) executa softwares operando em conjunto, como LEMP stack, Nginx, DNS BIND (resolução de nomes em endereços IP), GeoIP (filtro geográfico). Por exemplo, um visitante da Alemanha recebe o IP do nó de borda localizado no local mais próximo na Europa, dependendo dos filtros configurados com o GeoIP.

O software Nginx é um servidor Web capaz de lidar com tráfego intenso de maneira consistente. A ideia é aproveitar os recursos de proxy e armazenamento em cache. Para isso, é necessário compilar o Nginx e alavancar o módulo proxy. Este módulo permite armazenar dados em cache nos discos locais dos pontos de presença remotos. Para auxiliar na criação de caches, o Varnish Cache é um mecanismo de armazenamento de alto desempenho usado para manter o conteúdo acessado recentemente na memória. Existe a necessidade de instalar o Varnish para operar em conjunto com o Nginx, que agirá como um servidor de conteúdo nos nós de borda. Essa CDN mantém a última cópia de arquivos acessados mais próxima dos visitantes globais e também armazena em cache as páginas mais utilizadas na memória dos nós de borda. Isso significa menos tempo de acesso aos recursos da rede pelos usuário.

Os equipamentos utilizados são descritos na Tabela 1. Um nó está localizado na cidade de S. Rita do Sapucaí/MG no Inatel e o outro na cidade de Hortôlandia/SP. A arquitetura compreende servidores com alta capacidade de processamento e um enlace real de

Tabela 1. Características dos recursos de hardware da arquitetura proposta.

\begin{tabular}{|c|c|c|}
\hline Característica: & Descrição: & Localização: \\
\hline Servidor 1 & $\begin{array}{l}\text { DELL PowerEdge R540 Intel Xeon Silver } 4110 \text { CPU } 2.33 \text { GHz } 16 \text { Núcleos; } 64 \\
\text { GB de memória RAM; } 2 \text { SSDs } 480 \text { GB; } 6 \text { interfaces de } 10 \text { Gigabits. }\end{array}$ & $\begin{array}{l}\text { ICT Lab, Inatel St. } \\
\text { Rita Sapucaí/MG }\end{array}$ \\
\hline Servidor 2 & $\begin{array}{l}\text { DELL Intel Core i7-4790 CPU } 3.60 \text { GHz } 4 \text { Núcleos; } 16 \text { GB de memória RAM; } \\
\text { HD } 2 \text { TB; } 2 \text { interfaces de } 10 \text { Gigabits. }\end{array}$ & Hortolândia/SP \\
\hline Satélite SES-14 & Posição Orbital 47.5 W. Transponders operam nas bandas C, KU e feixes HTS. & Órbita GEO \\
\hline Modem 5G & $\begin{array}{l}\text { Desenvolvido no Inatel pelo } \text { CRR (Centro de } \\
\text { Radiocomunicações) } \\
\text { com Transceptores Flexíveis MIMO-GFDM. }\end{array}$ & ICT Lab, Inatel \\
\hline
\end{tabular}

Tabela 2. Características dos recursos de software da arquitetura proposta.

\begin{tabular}{|c|c|c|}
\hline Software: & Descrição: & Localização: \\
\hline GNU/Linux Ubuntu & $\begin{array}{l}\text { Versão } 16.04 \text { LTS para os servidores com hardwares físicos e versão } 18.04 \text { LTS } \\
\text { instalados nas VMs. }\end{array}$ & Servidor 1 e 2 \\
\hline Máquinas Virtuais & Virtual Machines (VMs) Kernel-based Virtual Machine (KVM). & Servidor 1 e 2 \\
\hline Nginx & Versão HTTP 2019-07-23 nginx-1.17.2. & Servidor 1 e 2 \\
\hline Varnish & $\begin{array}{l}\text { Versão 2019-03-15 - Varnish 6.2.0, instalado como mecanismo de armazena- } \\
\text { mento em cache de alto desempenho. }\end{array}$ & Servidor 1 \\
\hline LEMP Stack & $\begin{array}{l}\text { Versão SO Ubuntu Server 18.04.2 LTS, Nginx HTTP, MySQL Community Serve } \\
\text { 8.0.16 e PHP 7.3.7, são softwares de código aberto operando de forma conjunta } \\
\text { para criação de sites, gerenciamento de banco de dados relacional e linguagem } \\
\text { de programação para aplicativos da Web. }\end{array}$ & Servidor 2 \\
\hline BIND DNS & $\begin{array}{l}\text { Versão 9.14.4, instalado para publicar informações de DNS na Internet e resolver } \\
\text { consultas DNS para os hosts da rede, é a ferramenta que mantém todos esses } \\
\text { componentes se comunicando na rede, serve a cada cliente o IP do nó de borda } \\
\text { mais próximo na CDN. }\end{array}$ & Servidor 2 \\
\hline GeoIP & $\begin{array}{l}\text { Versão geoip_1.6.12, utilizado em servidores para buscar localizações baseadas } \\
\text { em IP. Com a utilização do GeoIP e do endereço IP do host, é possível localizar } \\
\text { a cidade, estado, país e até mesmo a latitude e longitude do endereço que está } \\
\text { acessando o conteúdo. }\end{array}$ & Servidor 2 \\
\hline
\end{tabular}


comunicação via satélite SES-14 para conexão entre os pontos de testes. As máquinas virtuais (VMs) contêm softwares que operam em conjunto para construir a topologia CDN. As VMs são criadas com o hypervisor KVM que é implementado diretamente no Kernel do sistema hospedeiro para otimizar a comunicação das máquinas virtuais com o hardware. Para a distribuição de conteúdos, são utilizados Proxies, Cache, plataformas Edge e Cloud Computing. Os recursos de software utilizados na implementação na arquitetura de testes são descritos na Tabela 2.

\section{Avaliação Experimental de Desempenho}

Nessa seção apresentamos a configuração do cenário de testes utilizado para avaliar o desempenho da solução proposta de distribuição de conteúdo com cache de rede em 5G híbrido. Foram utilizados dois nós: (i) nó de conteúdo dinâmico (ICT_SAT_5G_centralcdn-node) localizado na cidade de Hortolândia/SP no Servidor 2; e (ii) nó de localização de borda (ICT_SAT_5G_edgecdn-node) localizado no ICT Lab (Inatel) no Servidor 1. Inicialmente foi preciso configurar o Servidor 1. Dentro do programa $K V M Q E M U$ foram criadas duas máquinas que emulam o hardware real para instalação dos programas e ferramentas necessários para a execução dos testes. As configurações de cada nó são apresentadas na Tabela 3.

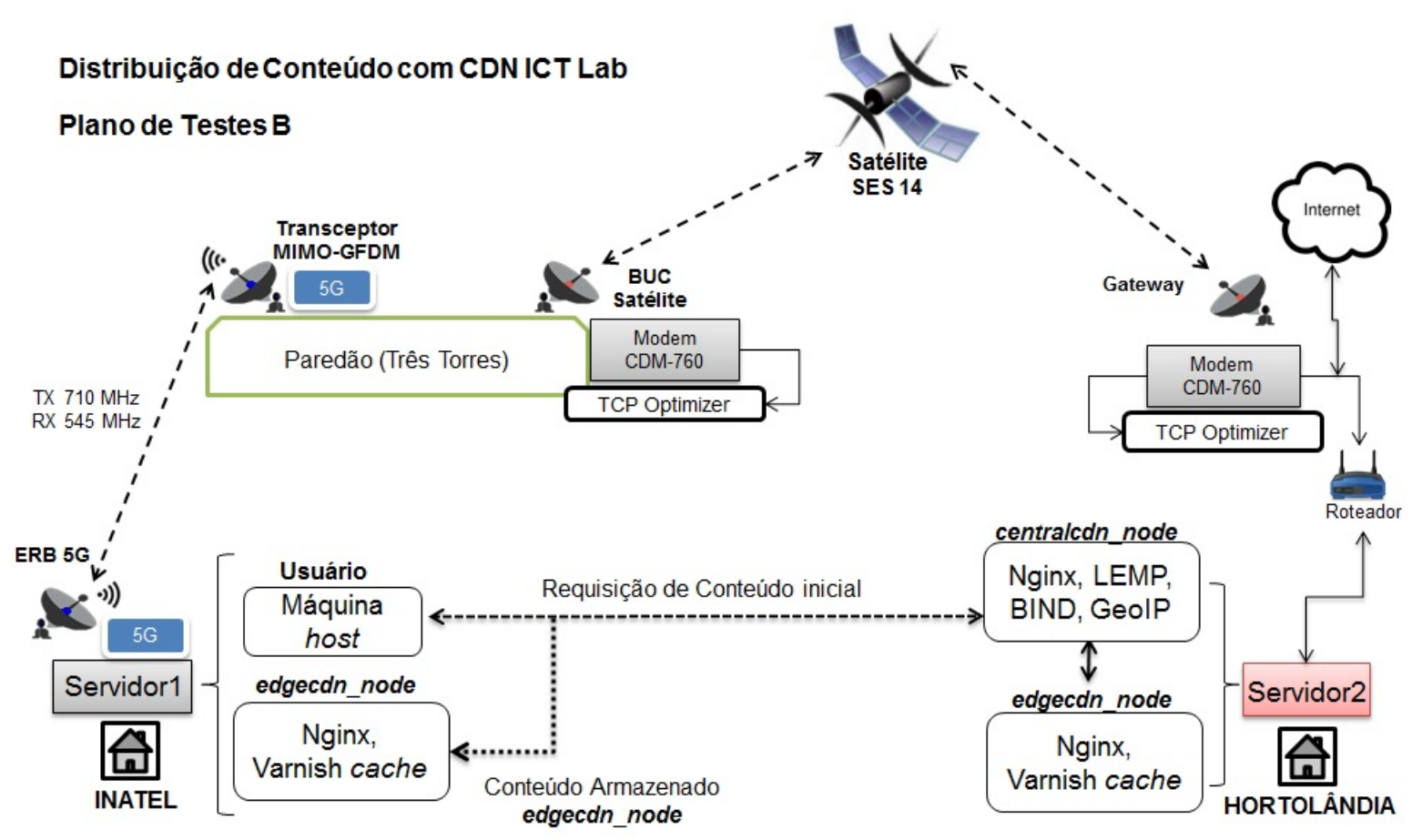

Figura 2. Configuração de testes de distribuição de conteúdo via 5G híbrido entre o ICT Lab (Inatel) Sta. Rita Sapucaí/MG e a estação de Hortolândia/SP.

O cenário de teste completo é apresentado na Figura 2. O Servidor 1 faz papel de usuário na rede e funciona como um host rodando uma cópia do nó de borda para as requisições de dados e mídia. O Servidor 2 roda uma cópia do nó central e do nó de borda, caso fosse necessário inverter o papel de usuário. O testes foram inicialmente configurados para conteúdo estático, através de uma página HTML com uma imagem padrão. Assim que todos os componentes foram instalados, foi possível realizar a configuração 
do protocolo IP em cada dispositivo da rede. O foco dos testes foi medir a latência durante a transferência de conteúdos, tais como imagens via enlace com o SES-14 e através do enlace terrestre formado por dois Modems 5G. Os programas utilizados para análise foram: WireShark, Nload, IPtraf, Nethogs, IFtop e MatLab.

As Tabelas de Testes 4 e 5 apresentam em detalhes os testes efetuados. Foram considerados trinta ensaios com recebimento e envio de dados para usuários visando a utilização de serviços de armazenamento estáticos e dinâmicos. Nas tabelas são informados o Tamanho do Arquivo transferido em Bytes, a Taxa Média de Transferência em Bytes por segundo e o Tempo de Transferência em segundos, obtido através da divisão do tamanho do arquivo pela taxa de transferência em cada ensaio. No final dos testes, foram calculados: (i) a Média dos Trinta Ensaios; (ii) a Taxa de Transferência; e (iii) a Razão entre o Tempo de Transferência quando se usa enlace via satélite e 5G apenas, dividido

Tabela 3. Características das máquinas virtuais utilizadas para experimentação.

\begin{tabular}{l|l} 
Característica: & Descrição: \\
\hline Processador & 2x Intel Core (Broadwell, IBRS) CPU 2.095 GHz 4 Núcleos x64 \\
Memórias & 2x 8 GB RAM \\
Placas de rede & 2x interface Gigabit 10/100/1000 Mbps e 1x interface de 10 Gigabits \\
Armazenamento & 1x HD 48 GB e 1x HD 80 GB \\
\hline
\end{tabular}

Tabela 4. Transferência de Conteúdo: Imagem .PNG sem CDN cache.

\begin{tabular}{l|l|l|l|l|l|l|l} 
& $\begin{array}{l}\text { Tamanho } \\
\text { (Bytes) }\end{array}$ & $\begin{array}{l}\text { Taxa (By- } \\
\text { tes/segundo) }\end{array}$ & $\begin{array}{l}\text { Tempo } \\
\text { (segundos) }\end{array}$ & & $\begin{array}{l}\text { Tamanho } \\
\text { (Bytes) }\end{array}$ & $\begin{array}{l}\text { Taxa (By- } \\
\text { tes/segundo) }\end{array}$ & $\begin{array}{l}\text { Tempo } \\
\text { (segundos) }\end{array}$ \\
\hline $\mathbf{1}$ & 3.083 .050 & 843.639 & 3,6545 & $\mathbf{1 6}$ & 3.083 .050 & 835.018 & 3,6922 \\
$\mathbf{2}$ & 3.083 .050 & 839.565 & 3,6722 & $\mathbf{1 7}$ & 3.083 .050 & 842.675 & 3,6586 \\
$\mathbf{3}$ & 3.083 .050 & 847.135 & 3,6394 & $\mathbf{1 8}$ & 3.083 .050 & 849.920 & 3,6275 \\
$\mathbf{4}$ & 3.083 .050 & 841.786 & 3,6625 & $\mathbf{1 9}$ & 3.083 .050 & 854.085 & 3,6098 \\
$\mathbf{5}$ & 3.083 .050 & 851.702 & 3,6199 & $\mathbf{2 0}$ & 3.083 .050 & 848.985 & 3,6315 \\
$\mathbf{6}$ & 3.083 .050 & 851.518 & 3,6207 & $\mathbf{2 1}$ & 3.083 .050 & 845.713 & 3,6455 \\
$\mathbf{7}$ & 3.083 .050 & 848.614 & 3,6330 & $\mathbf{2 2}$ & 3.083 .050 & 845.093 & 3,6482 \\
$\mathbf{8}$ & 3.083 .050 & 842.492 & 3,6594 & $\mathbf{2 3}$ & 3.083 .050 & 848.941 & 3,6316 \\
$\mathbf{9}$ & 3.083 .050 & 839.212 & 3,6737 & $\mathbf{2 4}$ & 3.083 .050 & 852.761 & 3,6154 \\
$\mathbf{1 0}$ & 3.083 .050 & 844.754 & 3,6496 & $\mathbf{2 5}$ & 3.083 .050 & 852.145 & 3,6180 \\
$\mathbf{1 1}$ & 3.083 .050 & 843.210 & 3,6563 & $\mathbf{2 6}$ & 3.083 .050 & 855.391 & 3,6043 \\
$\mathbf{1 2}$ & 3.083 .050 & 513.410 & 6,0050 & $\mathbf{2 7}$ & 3.083 .050 & 855.166 & 3,6052 \\
$\mathbf{1 3}$ & 3.083 .050 & 851.479 & 3,6208 & $\mathbf{2 8}$ & 3.083 .050 & 822.993 & 3,7461 \\
$\mathbf{1 4}$ & 3.083 .050 & 844.171 & 3,6522 & $\mathbf{2 9}$ & 3.083 .050 & 830.211 & 3,7136 \\
$\mathbf{1 5}$ & 3.083 .050 & 844.723 & 3,6498 & $\mathbf{3 0}$ & 3.083 .050 & 851.553 & 3,6205 \\
\hline
\end{tabular}

Tabela 5. Transferência de Conteúdo: Imagem .PNG via CDN cache.

\begin{tabular}{l|l|l|l|l|l|l|l} 
& $\begin{array}{l}\text { Tamanho } \\
\text { (Bytes) }\end{array}$ & $\begin{array}{l}\text { Taxa (By- } \\
\text { tes/segundo) }\end{array}$ & $\begin{array}{l}\text { Tempo } \\
\text { (segundos) }\end{array}$ & & $\begin{array}{l}\text { Tamanho } \\
\text { (Bytes) }\end{array}$ & $\begin{array}{l}\text { Taxa (By- } \\
\text { tes/segundo) }\end{array}$ & $\begin{array}{l}\text { Tempo } \\
\text { (segundos) }\end{array}$ \\
\hline $\mathbf{1}$ & 3.083 .050 & 20.998 .465 & 0,1468 & $\mathbf{1 6}$ & 3.083 .050 & 26.742 .638 & 0,1153 \\
$\mathbf{2}$ & 3.083 .050 & 16.384 .376 & 0,1882 & $\mathbf{1 7}$ & 3.083 .050 & 32.065 .645 & 0,0961 \\
$\mathbf{3}$ & 3.083 .050 & 29.616 .855 & 0,1041 & $\mathbf{1 8}$ & 3.083 .050 & 24.415 .634 & 0,1263 \\
$\mathbf{4}$ & 3.083 .050 & 25.216 .863 & 0,1223 & $\mathbf{1 9}$ & 3.083 .050 & 25.615 .746 & 0,1204 \\
$\mathbf{5}$ & 3.083 .050 & 22.284 .618 & 0,1383 & $\mathbf{2 0}$ & 3.083 .050 & 25.085 .153 & 0,1229 \\
$\mathbf{6}$ & 3.083 .050 & 25.184 .470 & 0,1224 & $\mathbf{2 1}$ & 3.083 .050 & 27.645 .512 & 0,1115 \\
$\mathbf{7}$ & 3.083 .050 & 28.884 .424 & 0,1067 & $\mathbf{2 2}$ & 3.083 .050 & 25.185 .268 & 0,1224 \\
$\mathbf{8}$ & 3.083 .050 & 24.816 .861 & 0,1242 & $\mathbf{2 3}$ & 3.083 .050 & 27.845 .334 & 0,1107 \\
$\mathbf{9}$ & 3.083 .050 & 26.716 .864 & 0,1154 & $\mathbf{2 4}$ & 3.083 .050 & 25.585 .345 & 0,1205 \\
$\mathbf{1 0}$ & 3.083 .050 & 25.916 .869 & 0,1190 & $\mathbf{2 5}$ & 3.083 .050 & 38.345 .438 & 0,0804 \\
$\mathbf{1 1}$ & 3.083 .050 & 23.325 .291 & 0,1322 & $\mathbf{2 6}$ & 3.083 .050 & 20.522 .749 & 0,1502 \\
$\mathbf{1 2}$ & 3.083 .050 & 25.024 .548 & 0,1232 & $\mathbf{2 7}$ & 3.083 .050 & 24.114 .436 & 0,1279 \\
$\mathbf{1 3}$ & 3.083 .050 & 21.515 .851 & 0,1433 & $\mathbf{2 8}$ & 3.083 .050 & 33.685 .246 & 0,0915 \\
$\mathbf{1 4}$ & 3.083 .050 & 24.913 .521 & 0,1238 & $\mathbf{2 9}$ & 3.083 .050 & 32.315 .517 & 0,0954 \\
$\mathbf{1 5}$ & 3.083 .050 & 25.345 .071 & 0,1216 & $\mathbf{3 0}$ & 3.083 .050 & 25.115 .591 & 0,1228 \\
\hline
\end{tabular}



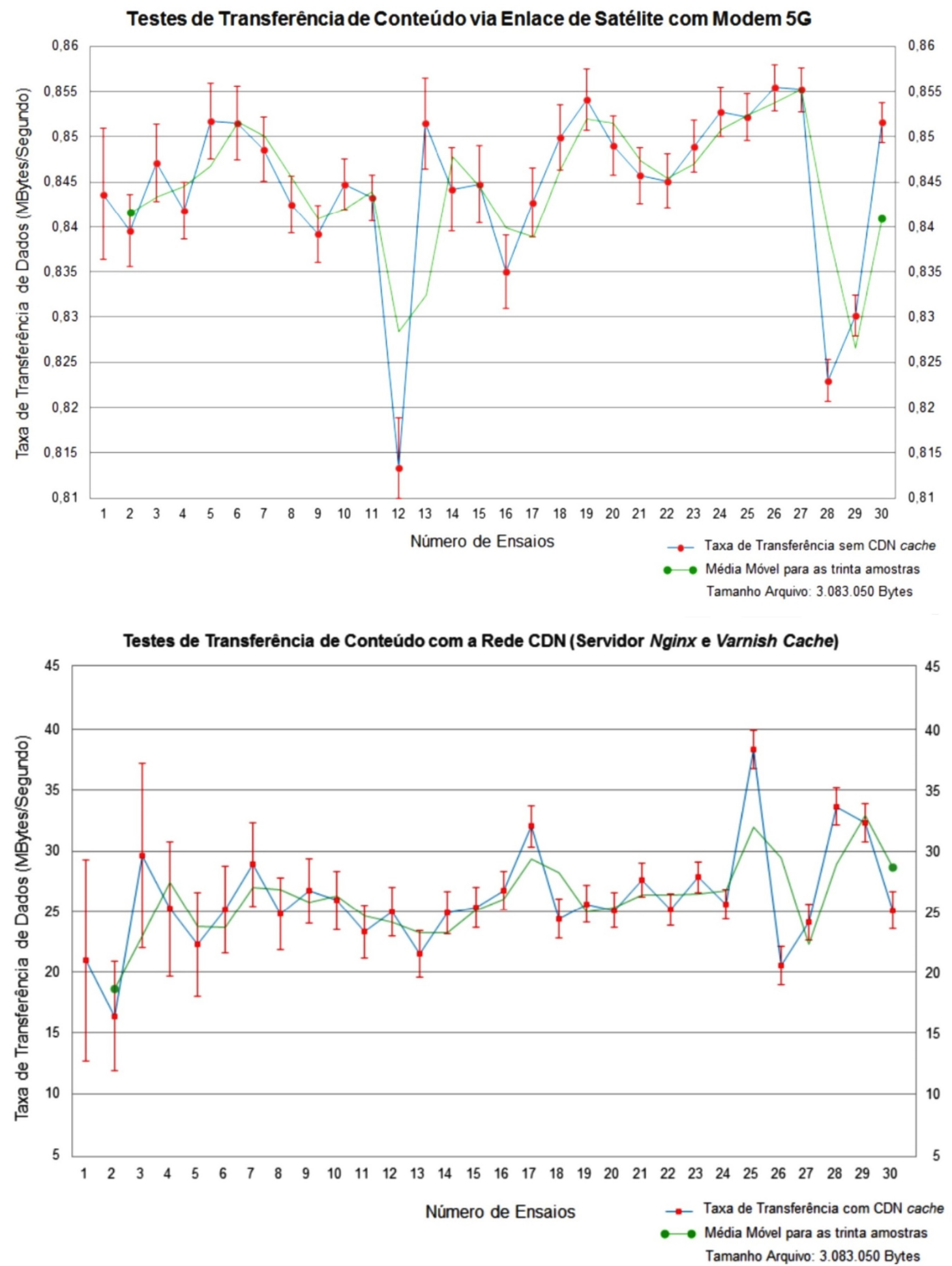

Figura 3. Análise da Taxa de Transferência pelo Número de Ensaios.

pelo tempo necessário para transferir o mesmo arquivo usando CDN na rede. Os resultados mostram que para um arquivo de imagem do tipo .PNG de 3.083.050 Bytes, o tempo médio de transferência entre os pontos de transmissão e envio é de 3,72457 segundos. Via CDN cache o tempo médio é de 0,12153 segundos. O tempo de transferência foi aproximadamente 30,64733 vezes menor quando se utiliza CDN com cache local. 
A Figura 3 apresenta uma análise comparativa entre a taxa de transferência em cada um dos trinta ensaios sem o serviço da CDN com armazenamento em cache. A velocidade média dos trinta ensaios foi de 844.602 Bytes/segundo para um arquivo do tipo .PNG com tamanho de 3.083.050 Bytes. A Figura 3 também apresenta a análise dos resultados com a taxa de transferência de dados em cada um dos trinta ensaios com a rede operando com o serviço CDN cache. A taxa média durante a transferência dos trinta arquivos para este caso foi de aproximadamente 26.014.340 Bytes/segundo. Em comparação com a taxa média obtida com os mesmos testes sem o serviço CDN cache, de 834.602 Bytes/segundo, consegue-se uma taxa de transferência 30 vezes maior (razão exata de 31,16976 vezes). A medida de latência média obtida para o enlace satélite foi de 560,930 ms (da aplicação PING: RTD (Round Trip Delay) minímo = 510,916, médio $=560,930$, máximo $=629,949$ e Jitter $=46,065 \mathrm{~ms}$ ). Para 11.458 pacotes transmitidos foram recebidos com sucesso 11.435 pacotes, resultando em uma perda de $0,002 \%$ da quantidade total dos pacotes.

\section{Considerações Finais}

Essa pesquisa contribui com a implementação e configuração de uma arquitetura 5G híbrida terrestre/satélite para distribuição de conteúdos com cache de rede. Sistemas de comunicação 5G híbridos são uma solução promissora para estender e complementar o acesso em todas as áreas de cobertura. Os dados obtidos com o experimento provam que a rede híbrida operando com $\mathrm{CDN}$ se torna mais rápida, robusta e eficiente, apresentando perda mínima de pacotes durante os testes. Através das análises destaca-se que quanto maior o tamanho do arquivo transferido em Bytes, menor é a razão entre os tempos de transferência na rede híbrida comparando os serviços com e sem CDN. Depois que a transferência é concluída é possível replicar o conteúdo via rede local (rede terrestre) para inúmeros usuários localizados próximo do nó de borda que contém uma cópia idêntica do arquivo. Para arquivos menores os resultados mostram grandes vantagens, cerca de 30 vezes os tempos gastos com as transferências realizadas apenas via enlace satélite operando com o Modem 5G. O serviço CDN pode ser configurado para analisar as demandas mais populares e armazenar somente os conteúdos mais acessados em horários em que o usuário não estiver utilizando a rede. Parte da carga pode ser transferida via satélite com alto throughput em um tempo maior, e uma vez que o conteúdo original chegar ao servidor de borda, a rede CDN armazena os conteúdos mais requisitados através de análise e fornece de maneira transparente para o usuário uma cópia do conteúdo em um tempo menor. Testes da arquitetura com fluxo contínuo de dados streaming fazem parte das propostas de atividades futuras. O 5G híbrido certamente tirará proveito de tecnologias de virtualização, controle e distribuição de conteúdo, reduzindo custos de operação e latência na rede. Contudo, os resultados obtidos reforçam e comprovam que a integração da comunicação via satélite será essencial para o atendimento aos vários usuários em potencial que desejam acessar serviços $5 \mathrm{G}$ em áreas remotas ou não atendidas. As redes de acesso via satélite $5 \mathrm{G}$ trazem possibilidades de inovação e melhorias para a sociedade e viabilizam a prestação de serviço de provedores no âmbito mundial para diversos casos de uso. Destacam-se: novas formas de digitalizar operações nos setores público e privado, impacto ambiental reduzido, segurança pública, Health Care, realidade virtual e aumentada, cidades inteligentes, setor industrial, veículos autônomos, dispositivos vestíveis, Artificial Intelligence (AI) e ambientes sempre conectados. 


\section{Agradecimentos}

Trabalho parcialmente financiado pelo Sindicato Nacional das Empresas de Telecomunicações por Satélite (SINDISAT). Também parcialmente financiado pela RNP, com recursos do MCTIC, processo N. 01250.075413/2018-04, sob o projeto CRR do Inatel, Brasil. Os autores agradecem também a FAPEMIG, CNPq e CAPES.

\section{Referências}

3GPP (2016). $\operatorname{Tr}$ 38.913. In Release 14. [Online: 12/12/2015] Disponível em: http://www.3gpp.org/DynaReport/38913.htm. Acesso em: 19 Jul 2018 às 13:40.

A. Guidotti, A. Vanelli-Coralli, S. C. (2018). Archit. and key technical chall. for 5g sys. incor. satellites. In Trans. on Vehic. Technologies.

C. Young, J. Kim, N. P. (2016). Revolutionary direction for $5 \mathrm{~g}$ mobile core network architecture. In Inter. Conf. on Inf. and Commun. Tech. Convergence. IEEE.

Dai, B. (2018). Prosp. of 5g commun. mode for energy internet. In 2nd IEEE Conf. on Ener. Int. and Ener. Sys. Integration. IEEE.

Evans, B. G. (2015). The rol. of satell. in 5g. In Adv. Sat. Multime. Syst. Conf. Signal Process. Sp. Commun. Work., volume 14, pages 197-202.

Frangoudis, L. Yala, A. K. (2017). Cdn-as-a-service provision over a telecom operator's cloud. In IEEE Trans. on Net. and Serv. Manag., volume 14, pages 702-716.

IEEE (2016). Stand. news. In IEEE Commun. Magazine, volume 54, pages 12-13. IEEE.

J. Min, X. Gu, N. Z. (2016). Broad. hybr. satell.-terrest. commun. sys. based on cognit. radio toward 5g. In IEEE Wir. Communications, volume 23, pages 96-106. IEEE.

K. Liolis, A. Geurtz, N. C. (2018). Use cas. scen. of 5g integ. satell.-terrest. net. enhan. mob. broad.: The sat5g approach. Int. Jour. Satell. Commun. Networking, Maio.

M. Luglio, S. Romano, F. Z. (2019). Serv. deliv. mod. conver. satell.-terres $5 \mathrm{~g}$ net deploy.: A satell.-assis. cdn use-case. In IEEE Network, volume 33, pages 142-150. IEEE.

Marcus, M. J. (2015). 5g and "imt for 2020 and beyond"spectrum pol. and regul. issues. In IEEE Wir. Communications, volume 22, pages 2-3. IEEE.

N. Panwar, S. Sharma, K. S. (2016). A sur. on 5g: The nex. gener. of mob. communication. In Phys. Commun., volume 18, pages 68-84.

N. Wang, I. Selinis, e. a. (2019). Qoe-assured live stream. via satell. backhaul in $5 \mathrm{~g}$ networks. In IEEE Trans. on Broadcasting, volume 65, pages 381-391. IEEE.

Naser, O. Y. A. (2017). Tech. for 5g net.: Challenges and opportunities. In IEEE IT Professional, volume 19.

SaT5G (2018). Sat5g project h2020. In SaT5G. [Online]: 01/06/2017 Disponível em: http://sat5g-project.eu/public-deliverables/. Acesso em: 05 Jul 2018 às 17:01.

W. Ejaz, W. W. (2016). Iot in 5g wirel. commun. IEEE Access, 4:10310-10314.

Y. Kawamoto, H. Nishiyama, Z. F. (2013). Effec. data collec. via satell.-routed sens. sys. to realize glob.-scal. iot. In IEEE Sens. Journal, volume 13, page 3645-3654.

Yang, Hewu Li, Q. W. (2018). An integ. unic. and multic. solution by ext. cont. deliv. net. to satellite. In 14th Inter. Wirel. Commun. and mob. Comp. Conference. IEEE. 